\title{
Anharmonic Effect on Unimolecular Reactions with Application to the Photodissociation of Ethylene $^{\dagger}$
}

\author{
L. Yao* \\ Department of Physics, Dalian Maritime University, Dalian 116023, China
}

\author{
A. M. Mebel \\ Department of Chemistry and Biochemistry, Florida International University, Miami, Florida 33199
}

\author{
H. F. Lu \\ Institute of Atomic and Molecular Sciences, Academia Sinica, P.O. Box 23-166, Taipei 10764, Taiwan
}

\author{
H. J. Neusser \\ Institute of Physical and Theoretical Chemistry, Technical University of Munich, Garching, 84748, Germany
}

\author{
S. H. Lin \\ Institute of Atomic and Molecular Sciences, Academia Sinica, P.O. Box 23-166, Taipei 10764, Taiwan, and \\ Department of Applied Chemistry, National Chiao-Tung University, Hsin-chu, Taiwan
}

Received: December 29, 2006; In Final Form: March 28, 2007

\begin{abstract}
The importance of anharmonic effect on dissociation of molecular systems, especially clusters, has been noted. In this paper, we shall present a theoretical approach that can carry out the first principle calculations of anharmonic canonical and microcanonical rate constants of unimolecular reactions within the framework of transition state theory. In the canonical case, it is essential to calculate the partition function of anharmonic oscillators; for convenience, the Morse oscillator potential will be used for demonstration in this paper. In the microcanical case, which involves the calculation of the total number of states for the activated complex and the density of states for the reactant, we make use of the fact that both the total number of states and the density of states can be expressed in the inverse Laplace transformation of the partition functions and that the inverse Laplace transformation can in turn be carried out by using the saddle-point method. We shall also show that using the theoretical approach presented in this paper the total number of states and density of states can be determined from thermodynamic properties and the difference between the method used in this paper and the thermodynamic model used by Krems and Nordholm will be given. To demonstrate the application of our theoretical approach, we chose the photodissociation of ethylene at 157 and $193 \mathrm{~nm}$ as an example.
\end{abstract}

\section{Introduction}

The present investigations focus on the quantitative examination of the anharmonic effect on the dissociation of molecules and the interpretation and analysis of the observed kinetic results from the viewpoint of the first principle calculations. In unimolecular reactions under collision-free conditions, according to the RRKM (Rice-Ramsperger-Kassel-Marcus) theory (or quasiequilibrium theory), the total number of states and density of states plays an important role in the calculation of the rate constants. To describe the anharmonic effect, the Morse oscillators (MOs) are commonly used. Recent reviews of the anharmonic effect on unimolecular reactions can be found in refs $1-12$. Some vibrational modes in molecules or clusters ${ }^{5}$ correspond to relatively weak bonds; in this case, the Morse potential can be conveniently used to fit the PES (potential

\footnotetext{
† Part of the special issue "M. C. Lin Festschrift".

* To whom correspondence should be addressed. E-mail: yaoli@ newmail.dlmu.edu.cn.
}

energy surface $)^{2}$ and to simulate the bonding. Typical features of the anharmonic effect include a decrease in vibrational bondstretching frequencies (a red shift) and an increase in the bond lengths and distance of bond dissociation., 3a An important type of anharmonicity investigated by Bhuiyan and Hase ${ }^{4 b}$ is the decrease in the bending frequency as a bond defining that the bend is stretched. For the $\mathrm{H}-\mathrm{C}-\mathrm{C}$ model triatomic, these authors found that at $90 \mathrm{kcal} / \mathrm{mol}$ the bend-stretch anharmonic correction is 1.81 and the MO anharmonic correction is 1.63 , for a total correction of 2.95. For separable systems, a state counting technique has been elaborated by Beyer and Swinehart using the Beyer-Swinehart algorithm for harmonic oscillators (HOs) and extended by Stein and Rabinovitch to anharmonic separable systems. ${ }^{6 a, b}$ The anharmonic shift of energy, the anharmonic correction factor, and the anharmonic vibrational mode have often been used to calculate the total number of states and density of states of the system. ${ }^{6-9}$

Recent studies ${ }^{4,5}$ have shown that anharmonic effects have become very important in clusters and macromolecules. The 
anharmonic force field calculations have been carried out. ${ }^{6}$ The need for anharmonic correction to existing reaction rate theories has been emphasized by several authors, ${ }^{7-9}$ and Troe ${ }^{10}$ proposed a simple empirical method for generating anharmonic vibrational densities of states using experimental thermodynamic data.

Recent rapid developments in quantum chemistry calculations have made it possible to perform the first principle calculations of reaction rate constants within the framework of the transition state theory. However, in unimolecular reactions, most of these calculations are still limited to the $\mathrm{HO}$ approximation. It is thus desirable to develop an elementary systematic theoretical approach that can be easily used to treat the anharmonic effect in both canonical and microcanonical unimolecular reactions.

For this purpose, in this paper, we present a method that can compute the total numbers of states, densities of states, partition functions, and rate constant by using the Morse potential as an example of an anharmonic potential, which in turn is determined by ab initio calculations. This can be accomplished by using the inversion of partition function (IOPF) $\operatorname{method}^{11,12}$ or the Darwin-Fowler method ${ }^{3}$ for calculating the number and density of states of the system. However, in this paper, the IOPF method will be used. Although the anharmonic effects have been studied by using the IOPF and Darwin-Fowler methods, ${ }^{11,12}$ the detailed calculations are different from those to be presented in this paper. A main purpose of the present paper is to discuss how to make use of anharmonic potential surfaces obtained from $\mathrm{ab}$ initio calculations to treat the anharmonic effects on both canonical and microcanonical unimolecular reactions within the framework of the transition state theory. We shall show that the central point of the approach to be presented in this paper is the calculation of partition functions, which can then be applied to canonical unimolecular reactions and to microcanonical unimolecular reactions by using the IOPF method. It should be noted that using a personal computer, Pentium 4 (775 $506 \mathrm{E} / 2.66 \mathrm{G})$, for a system of seven vibrational modes, it will take only about $10 \mathrm{~s}$ to obtain the total number of states and density of states by using the IOPF method, while using the exact counting method, it will take about 1 day; the computation time increases rapidly with the size of the system. Furthermore, the accuracy of our calculations can be within $1 \%$ if the second-order approximation of the saddle-point method is used. To demonstrate how we can perform the first principle calculation of anharmonic unimolecular reaction rate constants by combining this approach with ab initio calculations of anharmonic oscillators, we chose the photodissociation of ethylene as an example. In 1927, Bates and Taylor ${ }^{13}$ began to study the physical insight into the ethylene decomposition. This subject has been studied subsequently by many chemists through theoretical and experimental investigations (for detailed discussion, see refs 14-30).

In our previous papers, ${ }^{31-36}$ we performed ab initio calculations of the PES for photodissociation of ethylene and obtained branching ratios of the dissociation products by using RRKM theory in the harmonic oscillator (HO) approximation. In this paper, the anharmonic effect will be considered.

The present paper is organized as follows. In section 2, we present the theoretical treatment of the anharmonic effect on canonical and microcanonical unimolecular reactions, which will be followed by a numerical demonstration (see section 3 ). The theoretical application to ethylene is given in section 4 .

\section{Theory of Unimolecular Reactions and Anharmonic Effect}

2.1. Microcanonical Case. Dissociation of molecules is an important type of chemical reaction that has been widely studied using transition state theory. For treating microcanonical unimolecular reactions, the RRK theory was developed by Rice and Ramsperger ${ }^{37}$ in 1927 and Kassel ${ }^{38}$ in 1928. By taking the transition state theory developed by Eyring ${ }^{39}$ in 1935 into account, the RRK theory was generalized into the RRKM theory in 1952 by Marcus. ${ }^{40}$ The theory assumes that intramolecular vibrational energy redistribution occurs on a time-scale much faster than the unimolecular reaction. This method enables us to compute the unimolecular reaction rates from a few characteristics of the PES. Despite the consensus on the applicability of the RRKM theory, the possible role of restricted vibrational energy flow in modifying the rate of unimolecular reactions has resurfaced many times, and it has also been improved by a number of groups. ${ }^{41-46}$

For a microcanonical system, according to the RRKM theory, ${ }^{24}$ the unimolecular rate constant is given by

$$
k_{u}(E)=\frac{1}{h} \cdot \frac{W^{\neq}\left(E-E_{\mathrm{a}}^{\neq}\right)}{\rho(E)}
$$

where $h$ is Planck's constant, $\rho(E)$ is the density of the states of the unimolecular reactant, and $W^{\neq}\left(E-E_{\mathrm{a}}^{\neq}\right)$is the total number of states of the activated complex. Here, $E$ and $E_{\mathrm{a}}^{\neq}$ represent the total energy given to the system and the activation energy, respectively. Thus, in essence, as is expected in a statistical treatment, the RRKM theory simply states that the rate constant is proportional to the fraction of molecules having an internal energy $E$ that are apt to react ${ }^{47-49}$ irrespective of the way that the molecule has been activated to that energy. It should be emphasized that conventionally $W^{\neq}\left(E-E_{\mathrm{a}}^{\neq}\right)$and $\rho(E)$ have been evaluated in the harmonic approximation. Notice that in general from the definition of $W(E)$, the total number of states can be expressed as ${ }^{11,12,49}$

$$
W(E)=\sum_{i} H\left(E-E_{i}\right)
$$

where $H\left(E-E_{i}\right)$ denotes the Heaviside function. In this case, energy levels $E_{i}$ are calculated explicitly and $W(E)$ can then be obtained by direct counting of those states, which are below or equal to $E$. From eq 2-2, we obtain ${ }^{11,12,49}$

$$
\int_{0}^{\infty} \mathrm{d} E e^{-\beta E} W(E)=\frac{Q(\beta)}{\beta}=L[W(E)]
$$

where $\beta=1 / k T, k$ is Boltzmann's constant, $T$ is the temperature of the system, and $Q(\beta)$ is the canonical partition function of the system. Similarly, we obtain, from the definition of the density of states, ${ }^{11,12,49}$

$$
\int_{0}^{\infty} \mathrm{d} E e^{-\beta E} \rho(E)=Q(\beta)=L[\rho(E)]
$$

That is, eqs 2-3 and 2-4 denote the Laplace transformation of $W(E)$ and $\rho(E)$, respectively.

In other words, $W(E)$ and $\rho(E)$ can be obtained from eqs 2-3 and 2-4 by inverse Laplace transformation, which in turn can be expressed in terms of contour integrals. The contour integrals involved in the inverse Laplace transformation can be evaluated by using the saddle-point method (or method of steepest descent). ${ }^{11,12,49}$ To the first-order approximation of the saddle- 
point method, for $W(E)$, we find

$$
W(E)=\frac{e^{\beta_{1}^{*} E} Q\left(\beta_{1}^{*}\right)}{\beta_{1}^{*}\left\{2 \pi\left[\frac{1}{\beta_{1}^{* 2}}+\left(\frac{\partial^{2}}{\partial \beta^{2}} \ln Q(\beta)\right)_{\beta^{*}{ }_{1}}\right]\right\}^{1 / 2}}
$$

where

$$
E=\frac{1}{\beta_{1}^{*}}-\left[\frac{\partial}{\partial \beta} \ln Q(\beta)\right]_{\beta^{*}{ }_{1}}
$$

and $\beta_{1}^{*}$ denotes the saddle-point value of $\beta$. Similarly, for $\rho(E)$, we have

$$
\rho(E)=\frac{e^{\beta_{2}^{*} E} Q\left(\beta_{2}^{*}\right)}{\left\{2 \pi\left[\frac{\partial^{2}}{\partial \beta^{2}} \ln Q(\beta)\right]_{\beta^{*}}\right\}^{1 / 2}}
$$

where

$$
E=-\left[\frac{\partial}{\partial \beta} \ln Q(\beta)\right]_{\beta_{2}}
$$

and $\beta_{2}^{*}$ denotes the saddle-point value of $\beta .{ }^{11,12,49}$ The secondorder approximation calculations have also been accomplished but will not be presented in this paper.

2.2. Canonical Case. For a canonical system, using the transition state theory, the rate constant for unimolecular reactions can be expressed as ${ }^{11,30,44,49}$

$$
k_{h}(T)=\frac{k T}{h} \cdot \frac{Q^{\neq}(T)}{Q(T)} e^{-\left(E_{\mathrm{a}}^{\neq} / k T\right)}
$$

where $Q(T)$ and $Q^{\neq}(T)$ represent the partition function for the reactant and the activated complex, respectively. In this case, we have

$$
Q^{\neq}(T)=\prod_{i}^{N-1} q_{i}^{\neq}(T) ; Q(T)=\prod_{i}^{N} q_{i}(T)
$$

where $N$ is the number of the vibration modes of the reactant and $q_{i}^{\neq}(T)$ and $q_{i}(T)$ are the vibrational partition function for the activated complex and reactant for each mode, respectively.

From the above discussion, we can see that the partition function $Q(\beta)$ plays a very important role in the calculation of both canonical and microcanonical rate constants. To consider the anharmonic effect on a unimolecular reaction, the anharmonic number and density of states for a system of coupled MOs take a particularly simple form. For the MO, we have

$$
E_{n_{i}}=\left(n_{i}+\frac{1}{2}\right) \hbar \omega_{i}-x_{i}\left(n_{i}+\frac{1}{2}\right)^{2} \hbar \omega_{i}
$$

where $x_{i}$ is the Morse parameter, $\omega_{i}$ is the frequency of the $i$-th vibrational mode, and $n_{i}$ is the vibration quantum number of the vibrational mode. The maximum value of $n_{i}$ is represented by $n_{i}(m)$. In other words, the anharmonic effect has two features: One is that the energy spacings are not equal, and the other is the existence of a maximum quantum number $n_{i}(m)$. It should be noted the anharmonic constants $x_{i}$ for various molecules can be determined by ab initio calculations.

We shall study the effect of the second feature first. The general case of anharmonic effect will be presented in the next section. For this purpose, we shall use the so-called truncated harmonic oscillator (THO), that is,

$$
q_{i}(T)=\sum_{n_{i}=0}^{n_{i}(m)} e^{-\left[\left(n_{i}+1 / 2\right) \hbar \omega_{i} / k T\right]}=\frac{1-e^{-\left[\left(n_{i}(m)+1\right) \hbar \omega_{i} / k T\right]}}{e^{\hbar \omega_{i} / 2 k T}-e^{-\left(\hbar \omega_{i} / 2 k T\right)}}
$$

which should be compared with the harmonic case

$$
q_{i}(T)_{H}=\frac{1}{e^{\hbar \omega_{i} / 2 k T}-e^{-\left(\hbar \omega_{i} / 2 k T\right)}}
$$

A main difference between the $q_{i}(T)$ and the $q_{i}(T)_{H}$ is that as $T$ $\rightarrow \infty, q_{i}(T) \rightarrow n_{i}(m)+1$ while $q_{i}(T)_{H} \rightarrow \infty$. In numerous cases, the reaction rate is relatively little affected by rotation. However, there are cases where rotational effects on the rate constants have been experimentally and theoretically demonstrated. ${ }^{43}$ So, for simplicity, only the vibrational degrees of freedom will be considered in this paper; the rotational contribution can easily be included.

2.3. Determination of $\rho(E)$ and $W(E)$ from Thermodynamic Properties. A thermodynamic method for calculating the molecular density of states has been proposed by Krems and Nordholm. ${ }^{1}$ A starting point of their method is to propose a function form for $\rho(E)$,

$$
\begin{aligned}
& \rho(E)=a(E-b)^{c} \quad E>b \\
& \rho(E)=0 \quad E<b
\end{aligned}
$$

where $a, b$, and $c$ are to be determined. They then calculate the corresponding canonical partition function

$$
Q(T)=\int_{0}^{\infty} \mathrm{d} E e^{-(E / k T)} \rho(E)=a(k T)^{c+1} c ! e^{-(b / k T)}
$$

Using the partition function $Q(T)$, various thermodynamic properties can be calculated, which can then be used to determine parameters $a, b$, and $c$. It should be noted that another functional form for $\rho(E)$ has been suggested by Song and Hase. ${ }^{7}$

A starting point of our approach is to notice that eq 2-8 basically describes the relation between the thermodynamic energy $E$ and the temperature $\beta^{*}=1 / k T^{*}$ through the partition function $Q\left(\beta^{*}\right)$. Next, we make use of the relation between Helmholtz free energy $A$ and the partition function

$$
A=-\frac{1}{\beta^{*}} \ln Q\left(\beta^{*}\right)
$$

Using eqs 2-8 and 2-16, we can easily obtain

$$
\rho(E)=\frac{\beta^{*} e^{S / k}}{\left(\frac{2 \pi C_{v}}{k}\right)^{1 / 2}}
$$

where $S$ and $C_{V}$ represent the entropy and heat capacity of the system, respectively. Equation 2-17 shows that $\rho(E)$ can be determined from thermodynamical quantifies. To evaluate $W(E)$, we can use $\rho(E)=\mathrm{d} W(E) / \mathrm{d} E$. Various other methods have been proposed; for example, Troe ${ }^{10}$ had used the phase-space method, while Hase and co-worker ${ }^{5,7}$ had calculated relative anharmonic density of states by the multiple histogram/Nosé ${ }^{5}$ dynamics method.

\section{Numerical Demonstration}

3.1. Microcanonical Case. For numerical computation, for convenience, we shall consider the coupled oscillator case; for 
example, for the HO case, we have

$$
Q(\beta)=\prod_{i=1}^{N} \frac{1}{1-e^{-\beta \hbar \omega_{i}}}
$$

It follows that

$$
W^{\neq}\left(E-E_{\mathrm{a}}^{\neq}\right)=\frac{e^{\beta_{\neq}^{*}\left(E-E_{\mathrm{a}}^{\neq}\right)} \prod_{i=1}^{N-1} \frac{1}{1-e^{-\beta \hbar \omega_{i}^{*}}}}{\left\{2 \pi\left[1+\beta_{\neq}^{* 2}\left(\frac{\partial^{2}}{\partial \beta^{2}} \ln Q\left(\beta_{\neq}\right)\right)\right]_{\beta_{\neq}^{*}}^{1 / 2}\right.}
$$

where

$$
E-E_{\mathrm{a}}^{\neq}=\frac{1}{\beta_{\neq}^{*}}+\sum_{i=1}^{N-1} \frac{\hbar \omega_{i}^{\neq}}{e^{\beta_{\neq}^{*} \hbar \omega_{i}^{\neq}}-1}
$$

and

$$
\rho(E)=\frac{e^{\beta^{*} E} \prod_{i=1}^{N} \frac{1}{1-e^{-\beta^{*} \hbar \omega_{i}}}}{\left\{2 \pi\left[\left(\frac{\partial^{2}}{\partial \beta^{2}} \ln Q\left(\beta^{*}\right)\right)\right]_{\beta^{*}}\right\}^{1 / 2}}
$$

where

$$
E=\sum_{i=1}^{N} \frac{\hbar \omega_{i}}{e^{\beta^{*} \hbar \omega_{i}}-1}
$$

Similarly, for the case of MO, we have

$$
Q(\beta)=\prod_{i=1}^{N} \sum_{n_{i}=0}^{n_{i}(m)} e^{-\beta E_{n_{i}}}
$$

and a similar expression for $Q^{\neq}(\beta)$. It should be noted that the classical phase space integral has been determined for low frequency intermolecular modes of the $\mathrm{Cl}^{-}-\mathrm{CH}_{3} \mathrm{Cl}$ ion-dipole complex to determine the anharmonic correction. ${ }^{30}$

To test the performance of the approximation method discussed in this paper, Tables 1 and 2 present the comparison of the HO case between the exact results, ${ }^{49}$ which are the exact harmonic results by direct count, and the first-order approximation results of the saddle-point method for cyclopropane and acetylene as a function of energy. The MO results of $W(E)$ for $x_{i}=0.01$ and $x_{i}=0.1$ are also given for comparison with the HO results. The real values of $x_{i}^{1}$ 's can be approximately determined by ab initio calculations.

As the energy $E$ increases, as expected, the $W(E)$ increases sharply for all results in Tables 1 and 2. Comparing the exact results and the saddle-point method results for the $\mathrm{HO}$ case, the uncertainty is less than $7 \%$ at the lowest energy and decreases to $0.12 \%$ with $E$ increasing from 10 to $150 \mathrm{kcal} / \mathrm{mol}$ in Table 1. The similar conclusion can be seen in Table 2 for acetylene; the uncertainty is decreased from 20 to $2 \%$ with energy increasing from 2.31 to $36.9 \mathrm{kcal} / \mathrm{mol}$. The agreement between the exact and the approximate results is satisfactory; the small difference is because of the first-order approximation
TABLE 1: Comparison of the Evaluation of $W(E)$ by the Exact and the Saddle-Point Method Results of the HO and the MO Cases with $x_{i}=0.01$ and 0.1 for Cyclopropane with Frequencies $\epsilon_{i}=3221(6), 1478(3), 1118(7), 879(3)$, and $750(2) \mathrm{cm}^{-1 a}$

\begin{tabular}{ccccl}
\hline & \multicolumn{4}{c}{$W(E)$ cyclopropane } \\
\cline { 2 - 5 }$E(\mathrm{kcal} / \mathrm{mol})$ & exact harmonic & harmonic & MO $x_{i}=0.01$ & MO $x_{i}=0.1$ \\
\hline 10 & 802 & 746 & 852 & 4856 \\
20 & $7.75 \times 10^{4}$ & $7.72 \times 10^{4}$ & $9.66 \times 10^{4}$ & $1.59 \times 10^{6}$ \\
30 & $2.69 \times 10^{6}$ & $2.68 \times 10^{6}$ & $3.63 \times 10^{6}$ & $1.08 \times 10^{8}$ \\
40 & $4.97 \times 10^{7}$ & $5.01 \times 10^{7}$ & $7.31 \times 10^{7}$ & $2.74 \times 10^{9}$ \\
50 & $6.12 \times 10^{8}$ & $6.19 \times 10^{8}$ & $9.70 \times 10^{8}$ & $3.58 \times 10^{10}$ \\
100 & $5.84 \times 10^{13}$ & $5.87 \times 10^{13}$ & $1.30 \times 10^{13}$ & $5.60 \times 10^{13}$ \\
150 & $3.00 \times 10^{15}$ & $3.00 \times 10^{15}$ & $9.59 \times 10^{15}$ & $4.02 \times 10^{14}$
\end{tabular}

${ }^{a}$ The exact harmonic value is determined by direct count, and the "harmonic" is the approximate harmonic value from the saddle-point method. Exact results are given in ref 49.

TABLE 2: Comparison of the Evaluation of $W(E)$ by the Exact and the Saddle-Point Method Results of the $\mathrm{HO}$ and the MO Cases with $x_{i}=0.01$ and 0.1 for Acetylene with Frequencies $\epsilon_{i}=612(2), 729(2), 1974(1), 3287(1)$, and $3374(1) \mathrm{cm}^{-1 a}$

\begin{tabular}{cllll}
\hline & \multicolumn{5}{c}{$W(E)$} \\
\cline { 2 - 5 }$E(\mathrm{kcal} / \mathrm{mol})$ & exact harmonic & harmonic & MO $x_{i}=0.01$ & MO $x_{i}=0.1$ \\
\hline 2.31 & 5 & 4 & 4 & 7 \\
4.61 & 15 & 15 & 16 & 39 \\
9.23 & 94 & 100 & 111 & 343 \\
13.8 & 387 & 402 & 468 & 1277 \\
18.5 & $1.21 \times 10^{3}$ & $1.25 \times 10^{3}$ & $1.51 \times 10^{3}$ & $3.36 \times 10^{3}$ \\
23.1 & $3.23 \times 10^{3}$ & $3.24 \times 10^{3}$ & $4.08 \times 10^{3}$ & $7.20 \times 10^{3}$ \\
27.7 & $7.34 \times 10^{3}$ & $7.47 \times 10^{3}$ & $9.79 \times 10^{3}$ & $1.33 \times 10^{4}$ \\
36.9 & $2.98 \times 10^{4}$ & $3.04 \times 10^{4}$ & $4.35 \times 10^{4}$ & $3.19 \times 10^{4}$
\end{tabular}

${ }^{a}$ The exact harmonic value is determined by direct count, and the "harmonic" is the approximate harmonic value from the saddle-point method. Exact results are given in ref 49.

in the saddle-point method used in this paper and also due to the fact that the saddle-point method is an asymptotic approximation method. Our second-order saddle-point approximation yields the results within 1\% accuracy even for the lowest energy. This shows that the saddle-point method is a good approximation method in analyzing the experimental data of unimolecular reactions. Next, we study the anharmonic effect on $W(E)$. Again, cyclopropane and acetylene have been used as examples (see Tables 1 and 2). The anharmonicity values of $x_{i}=0.01$ and $x_{i}=0.1$ of the MO have been used. From Tables 1 and 2, we can see that even for the $x_{i}=0.01$ case the anharmonic effect is significant, which can be as high as a factor of 3 for cyclopropane. For the energy range of $0.0-$ $150.0 \mathrm{kcal} / \mathrm{mol}$, however, the effect is only about $40 \%$ for acetylene. Next, we consider the case of $x_{i}=0.1$. For cyclopropane, the effect starts at a factor of 6 at $10 \mathrm{kcal} / \mathrm{mol}$, reaches the maximum at a factor of 60 at $E=50 \mathrm{kcal} / \mathrm{mol}$, and then decreases to a factor of 0.13 at $E=150 \mathrm{kcal} / \mathrm{mol}$. This dramatic decrease of $W(E)$ at $E=150 \mathrm{kcal} / \mathrm{mol}$ is due to the fact that $E$ has gone beyond the dissociation energy of low-frequency modes; in fact, the energy is enough to break all of the bonds, and in that case, the number of the states cannot increase as fast as in the $\mathrm{HO}$ case. Unlike HO, the MO has a maximum energy level, and the maximum value decreases with the increase of $x_{i}$. So, it is this reason why the $W(E)$ of the MO case increases more gently than that of the HO case for $x_{i}=0.1$. For acetylene, the anharmonic effect starts at a factor of 1.3 at $E=2.31 \mathrm{kcal} / \mathrm{mol}$, increases with $E$ to a maximum factor of 3 at $E=13.8 \mathrm{kcal} / \mathrm{mol}$, 


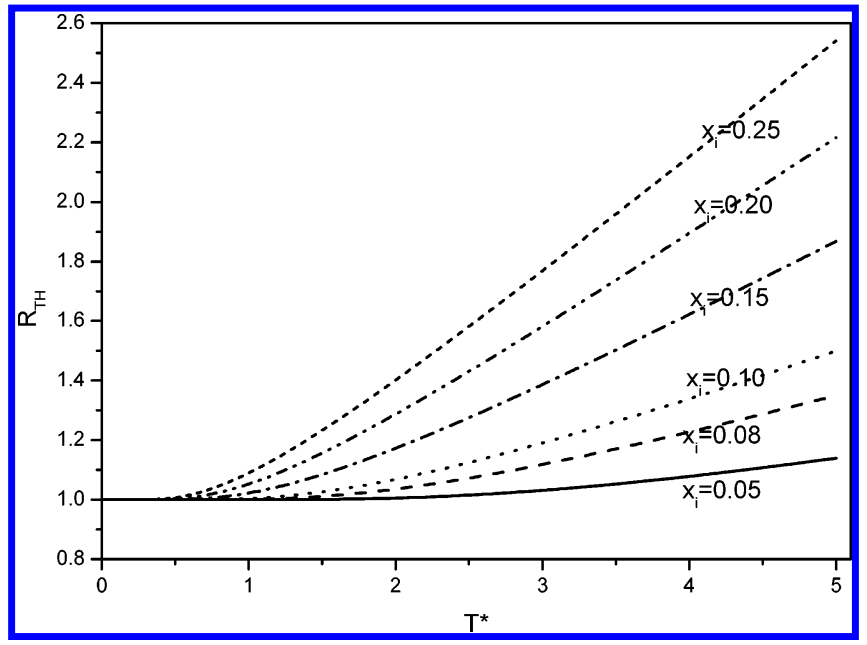

Figure 1. Plot of $R_{\mathrm{TH}}$ vs $T^{*}$ according to eq 3-14 for $x_{i}=0.05,0.08$, $0.10,0.15,0.20$, and 0.25 where the temperature $T^{*}$ as defined in eq $3-10$ is in the range of $0.01-5.0$ and is unitless. $R_{\mathrm{TH}}$, given by eq $3-14$, is the ratio of the rate constant of the THO to the HO.

and then decreases to the $\mathrm{HO}$ value at $E=36.9 \mathrm{kcal} / \mathrm{mol}$. From the above discussion, we can see that the anharmonic effect depends not only on the anharmonicity $x_{i}$ values but also on the size of molecules and the energy in the molecule.

It should be noted that the individual vibrational energy levels for $\mathrm{H}_{2} \mathrm{CO}$ and acetylene have been measured at high energies and the actual anharmonic density has been compared with the harmonic approximation. The anharmonic density is found to be 10 times larger for $\mathrm{H}_{2} \mathrm{CO}$ and six times larger for acetylene. These experimental studies have been reported. ${ }^{30}$

3.2. Canonical Case. For numerical calculations, for simplicity, we shall assume that ${ }^{11,30,44,49}$

$$
k_{u}(T)=\frac{k T}{h} \cdot \frac{1}{q_{i}(T)} e^{-\left(E_{\mathrm{a}}^{\neq} / k T\right)}
$$

In eq 3-7, it is assumed that except for the degree of freedom along the reaction coordinate, the vibrational properties for other degrees of freedom are the same for both reactant and activated complex. It is often more reasonable to include the zero-point energy (ZPE) in $E_{\mathrm{a}}^{\neq}$. In this case, we find for the $\mathrm{THO}$

$$
q_{i}(T)=\frac{1-e^{-\beta \epsilon_{i}\left[n_{i}(m)+1\right]}}{1-e^{-\beta \epsilon_{i}}}
$$

and

$$
k_{u}(T)_{T}=\frac{k T}{h} \cdot \frac{1-e^{-\beta \epsilon_{i}}}{1-e^{-\beta \epsilon_{i}\left[n_{i}(m)+1\right]}} e^{-\left(E_{\mathrm{a}} \neq / k T\right)}
$$

where $\epsilon_{i}=\hbar \omega_{i}$. In terms of reduced variables, defined by

$$
T^{*}=\frac{k T}{\hbar \omega_{i}} ;\left(E_{\mathrm{a}}^{\neq}\right)^{*}=\frac{E_{\mathrm{a}}^{\neq}}{\hbar \omega_{i}}
$$

and

$$
k_{u}^{*}\left(T^{*}\right)=\frac{2 \pi k_{u}(T)}{\omega_{i}}
$$

we obtain

$$
k_{u}^{*}\left(T^{*}\right)_{T}=\frac{T^{*}\left(1-e^{-\left(1 / T^{*}\right)}\right)}{1-e^{-\left(1 / T^{*}\right)\left[(1 / 2)+\left(1 / 2 x_{i}\right)\right]}} e^{-\left[\left(E_{\mathrm{a}}\right)^{*} / T^{*}\right]}
$$

which should be compared with the harmonic case,

$$
k_{u}^{*}\left(T^{*}\right)_{H}=T^{*}\left(1-e^{-\left(1 / T^{*}\right)}\right) e^{-\left[\left(E_{\mathrm{a}}\right)^{*} / T^{*}\right]}
$$

It follows that

$$
R_{T H}=\frac{k_{u}^{*}\left(T^{*}\right)_{T}}{k_{u}^{*}\left(T^{*}\right)_{H}}=\frac{1}{1-e^{-\left(1 / T^{*}\right)\left[\left(1 / 2 x_{i}\right)+(1 / 2)\right]}} \geq 1
$$

Next, we consider the MO case

$$
k_{u}^{*}\left(T^{*}\right)_{M O}=T^{*}\left(\sum_{n_{i}=0}^{n_{i}(m)} e^{-\left(1 / T^{*}\right)\left[n-x_{i}\left(n_{i}{ }^{2}+n_{i}\right)\right]}\right)^{-1} e^{-\left[\left(E_{\mathrm{a}}\right)^{*} / T^{*}\right]}
$$

and

$$
\begin{aligned}
R_{M O H}= & \frac{k_{u}^{*}\left(T^{*}\right)_{\mathrm{MO}}}{k_{u}^{*}\left(T^{*}\right)_{H}}= \\
& \left(1-e^{-\left(1 / T^{*}\right)}\right)^{-1}\left(\sum_{n_{i}=0}^{n_{i}(m)} e^{-\left(1 / T^{*}\right)\left[n_{i}-x_{i}\left(n_{i}^{2}+n_{i}\right)\right]}\right)^{-1}
\end{aligned}
$$

Results of numerical computation are shown in Figures 1 and 2.

In Figure 1, the ratio of the rate constants of the THO to the HO $R_{T H}$ is shown as the function of the temperature $T^{*}$ from 0.01 to 5.0. The ratio increases with anharmonic constant $x_{i}$ from 0.05 to 0.25 orderly with $T^{*}$ in the range of $0.01-5.0$. There is only a small difference between the rate constant of $\mathrm{HO}$ and that of the THO for $x_{i}=0.05$, while the difference becomes very pronounced when $x_{i}=0.25$, reaching a factor of 2. Figure 2 shows the behavior of the ratio of rate constants of MO case to $\mathrm{HO}$ case $R_{M O H}$ determined in eq 3-16 vs temperature $T^{*}$ for various $x_{i}$ values ranging from 0.01 to 0.25 ; below $T^{*}=$

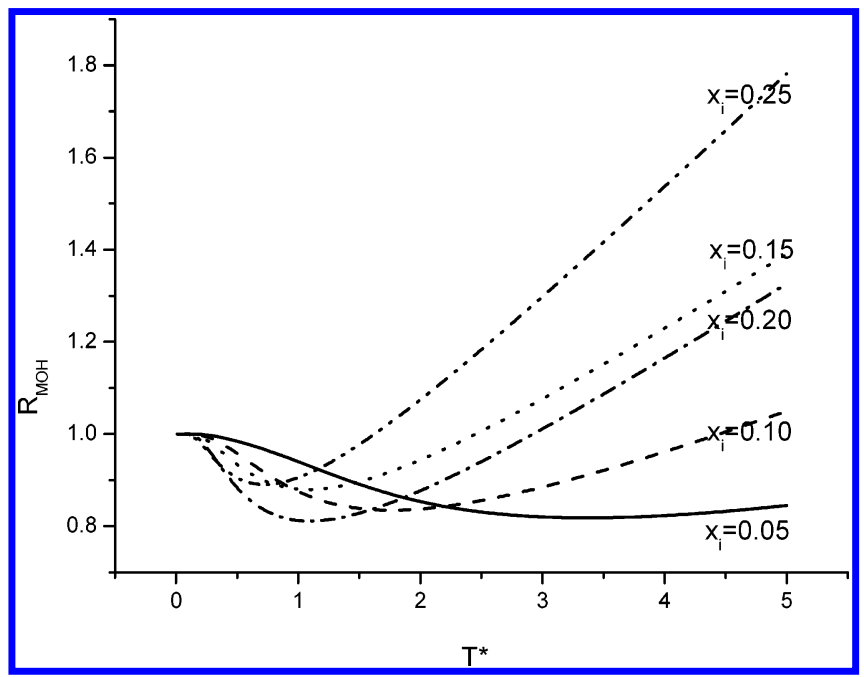

Figure 2. Plot of $R_{\mathrm{MOH}}$ vs $T^{*}$ according to eq 3-16 for $x_{i}=0.05$, $0.10,0.15,0.20$, and 0.25 . $R_{\mathrm{MOH}}$, given in eq $3-16$, is the ratio of the rate constant of the MO to the $\mathrm{HO}$ in the same temperature range as Figure 1 . 
TABLE 3: Harmonic Frequencies (in $\mathrm{cm}^{-1}$ ) of the Reactant, Transition States, and Intermediates for the $\mathrm{C}_{2} \mathrm{H}_{4}$ Dissociation Calculated at the B3LYP/6-311G** Level

\begin{tabular}{|c|c|c|c|c|c|c|c|c|c|c|}
\hline $\mathrm{C}_{2} \mathrm{H}_{4}$ & $\mathrm{CHCH}_{3}$ & $\mathrm{CHCH}_{2}$ & $\mathrm{H}_{2} \mathrm{CC}$ & TS1 & TS2 & TS4 & TS5 & TS7 & \multicolumn{2}{|c|}{$\mathrm{H} \cdots \mathrm{CHCH}_{2}$} \\
\hline 3136.9 & 3078.9 & 3235.8 & 3118.5 & $776.2 i$ & $1539.3 i$ & $631.5 i$ & $854.8 i$ & $590.5 i$ & $a$ & $b$ \\
\hline 1691.6 & 2987.3 & 3134.5 & 1710.4 & 3089.3 & 3239.5 & 3223.4 & 3368.7 & 3504.3 & $992.8 i$ & $447.9 i$ \\
\hline 1379.5 & 2902.3 & 3040.5 & 1210.7 & 2987.9 & 3136.3 & 3116.9 & 2486.2 & 3411.7 & 3276.1 & 3252.1 \\
\hline 1066.4 & 2830.8 & 1649.6 & 748.4 & 2953.7 & 2657.4 & 3056.2 & 1853.5 & 2005.8 & 3174.2 & 3144.8 \\
\hline 3121.7 & 1512.8 & 1391.1 & 3197.3 & 2255.8 & 1616.9 & 1683.7 & 923.5 & 820.8 & 3058.2 & 3042.5 \\
\hline 1472.0 & 1350.1 & 1045.8 & 337.2 & 1532.6 & 1383.9 & 1433.4 & 600.2 & 666.5 & 1659.3 & 1652.8 \\
\hline 972.7 & 1304.1 & 711.4 & & 1371.8 & 1043.5 & 1342.3 & & 369.9 & 1383.1 & 1387.9 \\
\hline 3221.6 & 1265.4 & 920.7 & & 1272.1 & 762.1 & 859.5 & & 767.1 & 1036.3 & 1034.6 \\
\hline 834.6 & 1118.8 & 819.2 & & 1259.7 & 1469.7 & 584.9 & & 645.4 & 760.0 & 711.3 \\
\hline 3193.2 & 959.4 & & & 1127.9 & 1007.0 & 886.7 & & & 470.9 & 189.1 \\
\hline 1238.7 & 613.3 & & & 1056.7 & 838.9 & 763.5 & & & 927.6 & 922.6 \\
\hline \multirow[t]{2}{*}{973.3} & 476.9 & & & 960.0 & 552.3 & 603.5 & & & 833.9 & 819.2 \\
\hline & & & & & & & & & 436.4 & 181.1 \\
\hline
\end{tabular}

${ }^{a}$ Geometry optimized at $\mathrm{C} \cdot \cdot \mathrm{H}=2.4 \AA .{ }^{b}$ Geometry optimized at $\mathrm{C} \cdot \cdots \mathrm{H}=3.0 \AA$.

TABLE 4: Anharmonic Constants $x_{i}\left(\right.$ in $^{-1}$ ) Corresponding to Table 3

\begin{tabular}{|c|c|c|c|c|c|c|c|c|c|c|}
\hline $\mathrm{C}_{2} \mathrm{H}_{4}$ & $\mathrm{CHCH}_{3}$ & $\mathrm{CHCH}_{2}$ & $\mathrm{H}_{2} \mathrm{CC}$ & TS1 & TS2 & TS4 & TS5 & TS7 & \multicolumn{2}{|c|}{$\mathrm{H} \cdots \mathrm{CHCH}_{2}$} \\
\hline 0.0214 & 0.0236 & 0.0224 & 0.0234 & 0.0210 & 0.0259 & 0.0243 & 0.0210 & 0.0179 & $a$ & $b$ \\
\hline 0.0085 & 0.0326 & 0.0253 & 0.0042 & 0.0292 & 0.0247 & 0.0241 & 0.0162 & 0.0175 & 0.0229 & 0.0225 \\
\hline 0.0086 & 0.0249 & 0.0272 & 0.0187 & 0.0285 & 0.0248 & 0.0485 & 0.013 & 0.0056 & 0.0231 & 0.0245 \\
\hline 0.0097 & 0.0251 & 0.0096 & 0.0152 & 0.0001 & 0.0190 & 0.0092 & 0.0017 & 0.0132 & 0.0180 & 0.0265 \\
\hline 0.0289 & 0.0141 & 0.0117 & 0.0259 & 0.0145 & 0.0144 & 0.0001 & 0.0087 & 0.0130 & 0.0094 & 0.0095 \\
\hline 0.0113 & 0.0178 & 0.0244 & 0.0923 & 0.0215 & 0.0222 & 0.0125 & & 0.0240 & 0.0083 & 0.0113 \\
\hline 0.0067 & 0.0154 & 0.0287 & & 0.0150 & 0.0099 & 0.0089 & & 0.0103 & 0.0167 & 0.0189 \\
\hline 0.0225 & 0.0137 & 0.0073 & & 0.0177 & 0.0001 & 0.0282 & & 0.0001 & 0.0128 & 0.0278 \\
\hline 0.0011 & 0.0115 & 0.0117 & & 0.0277 & 0.0133 & 0.0091 & & & 0.0001 & 0.0001 \\
\hline 0.0222 & 0.0091 & & & 0.0128 & 0.0001 & 0.0105 & & & 0.0074 & 0.0025 \\
\hline 0.0083 & 0.0990 & & & 0.0113 & 0.0116 & 0.0195 & & & 0.0099 & 0.0133 \\
\hline 0.0056 & 0.0710 & & & & & & & & 0.0001 & 0.0001 \\
\hline
\end{tabular}

${ }^{a}$ Geometry optimized at $\mathrm{C} \cdot \cdot \mathrm{H}=2.4 \AA .{ }^{b}$ Geometry optimized at $\mathrm{C} \cdot \cdot \mathrm{H}=3.0 \AA$.

2 , the anharmonic effect is not large, but the behavior is quite dramatic. From Figures 1 and 2, we can see that for temperature $T^{*}$ below 2 , the anharmonic effect for the models described by eqs 3-14 and 3-16 is not significant. This is because we are dealing basically with the anharmonic effect on a single vibrational mode case.

\section{Applications to Ethylene}

As reported in our previous papers, ${ }^{31-36}$ the ethylene photodissociation occurs via a vibrationally equilibrated hot ground state following internal conversion by hopping from one PES to another via the radiationless transition from an excited electronic state initially pumped through the excitation energy of 193 or $157 \mathrm{~nm}$.

The rate equations of the $\mathrm{C}_{2} \mathrm{H}_{4}$ photodissociation at 193 and $157 \mathrm{~nm}$ under the collision-free condition are given by

$$
\begin{aligned}
& \left\{\begin{array}{l}
\mathrm{C}_{2} \mathrm{H}_{4} \stackrel{k_{1}}{\stackrel{k_{-1}}{\longrightarrow}} \mathrm{CHCH}_{3} \\
\mathrm{CHCH}_{3} \stackrel{k_{2}}{\longrightarrow} \mathrm{HC} \equiv \mathrm{CH}+\mathrm{H}_{2}
\end{array}\right. \\
& \left\{\begin{array}{l}
\mathrm{C}_{2} \mathrm{H}_{4} \stackrel{k_{4}}{\longrightarrow} \mathrm{H}_{2} \mathrm{CC}:+\mathrm{H}_{2} \\
\mathrm{H}_{2} \mathrm{CC}: \stackrel{k_{5}}{\longrightarrow} \mathrm{HC} \equiv \mathrm{CH}
\end{array}\right. \\
& \left\{\begin{array}{l}
\mathrm{C}_{2} \mathrm{H}_{4} \stackrel{k_{6}}{\longrightarrow} \mathrm{CHCH}_{2}+\mathrm{H} \\
\cdot \mathrm{CHCH}_{2} \stackrel{k_{7}}{\longrightarrow} \mathrm{HC} \equiv \mathrm{CH}+\mathrm{H}
\end{array}\right.
\end{aligned}
$$

Two separate $\mathrm{H}_{2}$ elimination channels are inferred as follows: a 1,1-elimination producing the vinylidene radical in eq 4-2 and a 1,2-elimination producing the acetylene molecule in eq 4-1 and only one $\mathrm{H}$ loss channel in eq 4-3 with one-photon absorption considered in this paper (multiphoton absorption is neglected). ${ }^{14}$ We have applied the RRKM theory in the harmonic approximation to analyze the experimental data in the previous papers. ${ }^{31-36}$ In this paper, we shall study the anharmonic effect on these reactions. The ground state PES (including the anharmonic effect) of ethylene is obtained by ab initio calculations; transition states and isomers relevant to the primary dissociation channels eqs 4-1 to 4-3 are characterized in this paper. The rate constants of the ethylene photodissociation based on the calculated PES are computed by using the RRKM theory (including the anharmonic effect) at 193 and $157 \mathrm{~nm}$.

The geometry optimization and vibration frequencies of HOs and anharmonic oscillators of ethylene, intermediates, transition states, and products related to the possible dissociation channels were performed at the B3LYP/6-311G(d,p) level. ${ }^{50}$ The singlepoint energy calculation was carried out using the $\operatorname{CCSD}(\mathrm{T}) /$ $6-311+\mathrm{G}(3 \mathrm{df}, 2 \mathrm{p})$ method. ${ }^{51}$ The energies were corrected by $\mathrm{ZPE}$, either harmonic ZPE or anharmonic ZPE, to obtain the relative energies, corresponding to harmonic and anharmonic considerations. The rate constant of $\mathrm{H}$-elimination from ethylene is obtained by scanning the distance of $\mathrm{C}-\mathrm{H}$ via variation RRKM theory, which was described in our previous study. All $\mathrm{ab}$ initio calculations were carried out using the Gaussian 03 program package..$^{52}$

Previous electronic structure B3LYP/6-311G(d,p) calculations in our group have characterized the relative energies and transition states frequencies of ethylene. The dissociation reactions discussed below are the reactions that are energetically possible for ethylene when the molecule absorbs 193 or 157 nm photons. Thus, over most of the VUV range (but not at 193 $\mathrm{nm}$ ), we expect that two $\mathrm{H}$ atoms or $\mathrm{H}_{2}$ will be liberated. However, the $\mathrm{H}$ atoms should emerge sequentially, one from a hot ethylene and the other from a hot vinyl radical. 
TABLE 5: Relative Energies (in kcal/mol) of the Transition States and Intermediates for the $\mathrm{C}_{2} \mathrm{H}_{4}$ Dissociation Calculated at the CCSD(T)/6-311+G(3df,2p)//B3LYP/6-311G** Level (the First and Second Lines Are the Results Based on Harmonic and Anharmonic ZPE Values) ${ }^{a}$

\begin{tabular}{ccccccccrr}
\hline $\mathrm{C}_{2} \mathrm{H}_{4}$ & $\mathrm{CHCH}_{3}$ & $\mathrm{H}+\mathrm{CHCH}_{2}$ & $\mathrm{H}_{2} \mathrm{CC}+\mathrm{H}_{2}$ & TS1 & TS2 & TS4 & TS5 & TS7 & $\mathrm{H} \cdots \mathrm{CHCH}_{2}$ \\
\hline 0.0 & 73.33 & 107.16 & 82.11 & 74.56 & 107.30 & 93.79 & 83.25 & 145.69 & 93.37 \\
0.0 & 72.97 & 106.62 & 81.70 & 73.36 & 104.99 & 92.80 & 82.08 & 144.95 & 92.37
\end{tabular}

${ }^{a}$ The harmonic and anharmonic frequencies (in $\mathrm{cm}^{-1}$ ) of $\mathrm{H} \cdots \mathrm{CHCH}_{2}$ based on the distance of $\mathrm{H} \cdots \mathrm{C}$ at $2.4 \AA^{35}$ for the $\mathrm{C}_{2} \mathrm{H}_{4}$ dissociation calculated at the $\operatorname{CCSD}(\mathrm{T}) / 6-311+\mathrm{G}(3 \mathrm{df}, 2 \mathrm{p}) / / \mathrm{B} 3 \mathrm{LYP} / 6-311 \mathrm{G}^{* *}$ level.

TABLE 6: Number of States $W(E)$ and Density of States $\rho(E)$ for the Dissociation of Ethylene at 157 and $193 \mathrm{~nm} O b t a i n e d$ Using the Harmonic and Anharmonic Approximations

\begin{tabular}{|c|c|c|c|c|c|c|c|c|}
\hline \multirow[b]{3}{*}{$\mathrm{C}_{2} \mathrm{H}_{4}$} & \multicolumn{4}{|c|}{$W(E)$} & \multicolumn{4}{|c|}{$\rho(E)$} \\
\hline & \multicolumn{2}{|c|}{157} & \multicolumn{2}{|c|}{193} & \multicolumn{2}{|c|}{157} & \multicolumn{2}{|c|}{193} \\
\hline & harmonic & anharmonic & harmonic & anharmonic & harmonic & anharmonic & harmonic & anharmonic \\
\hline$k_{1}$ & $4.29 \times 10^{8}$ & $1.15 \times 10^{9}$ & $1.16 \times 10^{7}$ & $2.95 \times 10^{7}$ & $2.76 \times 10^{7}$ & $9.58 \times 10^{7}$ & $3.92 \times 10^{6}$ & $1.05 \times 10^{7}$ \\
\hline$k_{-1}$ & $3.36 \times 10^{10}$ & $2.68 \times 10^{11}$ & $4.56 \times 10^{9}$ & $2.63 \times 10^{10}$ & $1.09 \times 10^{8}$ & $1.33 \times 10^{8}$ & $1.51 \times 10^{7}$ & $1.71 \times 10^{7}$ \\
\hline$k_{2}$ & $3.49 \times 10^{10}$ & $2.10 \times 10^{11}$ & $3.08 \times 10^{9}$ & $1.28 \times 10^{10}$ & $1.09 \times 10^{8}$ & $1.33 \times 10^{8}$ & $1.51 \times 10^{7}$ & $1.71 \times 10^{7}$ \\
\hline$k_{4}$ & $4.65 \times 10^{8}$ & $1.50 \times 10^{9}$ & $7.92 \times 10^{6}$ & $1.92 \times 10^{7}$ & $2.76 \times 10^{7}$ & $9.58 \times 10^{7}$ & $3.92 \times 10^{6}$ & $1.05 \times 10^{7}$ \\
\hline$k_{6}{ }^{a}$ & $9.13 \times 10^{8}$ & $1.98 \times 10^{9}$ & $1.46 \times 10^{7}$ & $2.55 \times 10^{7}$ & $2.76 \times 10^{7}$ & $9.58 \times 10^{7}$ & $3.92 \times 10^{6}$ & $1.05 \times 10^{7}$ \\
\hline$k_{6}{ }^{b}$ & $5.59 \times 10^{9}$ & $1.47 \times 10^{10}$ & $8.58 \times 10^{7}$ & $1.82 \times 10^{8}$ & $2.76 \times 10^{7}$ & $9.58 \times 10^{7}$ & $3.92 \times 10^{6}$ & $1.05 \times 10^{7}$ \\
\hline
\end{tabular}

${ }^{a}$ Geometry optimized at $\mathrm{C} \cdot \cdot \mathrm{H}=2.4 \AA .{ }^{b}$ Geometry optimized at $\mathrm{C} \cdot \cdot \mathrm{H}=3.0 \AA$.

TABLE 7: Rate Constants of the $\mathrm{C}_{2} \mathrm{H}_{4}$ Dissociation Calculated Using Eqs 2-1 to 2-8 for the HO and MO Cases

\begin{tabular}{lccccc}
\hline & \multicolumn{2}{c}{$157 \mathrm{~nm}$} & & \multicolumn{2}{c}{$193 \mathrm{~nm}$} \\
\cline { 2 - 3 } \cline { 5 - 6 } $\mathrm{C}_{2} \mathrm{H}_{4}$ & harmonic & anharmonic & & harmonic & anharmonic \\
\hline$k_{1}$ & $4.65 \times 10^{11}$ & $3.61 \times 10^{11}$ & & $8.89 \times 10^{10}$ & $8.46 \times 10^{10}$ \\
$k_{-1}$ & $9.23 \times 10^{12}$ & $6.07 \times 10^{13}$ & & $9.07 \times 10^{12}$ & $4.62 \times 10^{13}$ \\
$k_{2}$ & $9.61 \times 10^{12}$ & $4.75 \times 10^{13}$ & & $6.12 \times 10^{12}$ & $3.00 \times 10^{13}$ \\
$k_{4}$ & $5.04 \times 10^{11}$ & $4.73 \times 10^{11}$ & & $6.06 \times 10^{10}$ & $5.50 \times 10^{10}$ \\
$k_{6}{ }^{a}$ & $9.90 \times 10^{11}$ & $6.19 \times 10^{11}$ & & $1.12 \times 10^{11}$ & $7.30 \times 10^{10}$ \\
$k_{6}{ }^{b}$ & $4.66 \times 10^{12}$ & $3.40 \times 10^{12}$ & & $5.09 \times 10^{11}$ & $5.22 \times 10^{11}$
\end{tabular}

${ }^{a}$ Geometry optimized at $\mathrm{C} \cdots \mathrm{H}=2.4 \AA .{ }^{b}$ Geometry optimized at $\mathrm{C} \cdot \cdot \mathrm{H}=3.0 \AA$.

The numerical results obtained for the photodissociation reactions based on the harmonic and anharmonic surfaces at 193 and $157 \mathrm{~nm}$ are given in Tables 3-7. All of the vibration modes have been treated as anharmonic MO; the harmonic frequencies of various species and corresponding anharmonic constants $x_{i}$ values, calculated by the Gaussian03 $03^{52}$ program package, have been chosen as effective dissociation energy parameters for the Morse potential in the calculations for each vibration mode.

Table 3 gives the harmonic frequencies of the reactant, transition states, and intermediates for the $\mathrm{C}_{2} \mathrm{H}_{4}$ dissociation, which are obtained from ab initio calculations for the channels in eqs 4-1 to 4-3, and to show the anharmonic effect, the $x_{i}$ values corresponding to Table 3 are given in Table 4. From Table 4 , we can see that the $x_{i}$ values are quite small for all modes. The relative energies of the transition states and intermediates for the $\mathrm{C}_{2} \mathrm{H}_{4}$ dissociation are given in Table 5 . Using the above data, in Table 6 , we have calculated the total number of states of the activated complexes and the density of states of the reactant and intermediates for the reactions described by eqs 4-1 to 4-3 by using the methods discussed in sections 2 and 3, for $\mathrm{HO}$ and MO at wavelengths of 193 and $157 \mathrm{~nm}$. From this table, we can see that the values of $W(E)$ and $\rho(E)$ for the MO case are always bigger than those of the $\mathrm{HO}$ case; because the energy levels $E_{i}$ in the MO are smaller than or nearly equal to the corresponding energy levels in the $\mathrm{HO}$, these results will introduce larger numbers of states and densities of states for the MO.

The rate constants for $k_{1}, k_{-1}, k_{2}, k_{4}$, and $k_{6}$ from eqs $4-1$ to 4-3 of the $\mathrm{C}_{2} \mathrm{H}_{4}$ dissociation are given in Table 7. $k_{5}$ and $k_{7}$ defined in eqs 4-1 to 4-3 are not included in this table, because we cannot easily determine the energy of the intermediates $\mathrm{H}_{2} \mathrm{CC}$ : and $\mathrm{CHCH}_{2}$ exactly. Furthermore, the present paper is focused only on the anharmonic effect on the rate constants rather than on the reaction mechanisms. So, the anharmonic effect on $k_{5}$ and $k_{7}$ is not included in our discussion. From Table 7 , we can see that in most cases, the anharmonic effect is significant. As expected, the anharmonic effect at $157 \mathrm{~nm}$ is in most cases more pronounced than that at $193 \mathrm{~nm}$. The computed anharmonic rate constants $k_{-1}$ and $k_{2}$ are in the range of $10^{13}$ $\mathrm{s}^{-1}$ by the order of magnitude, at 157 and $193 \mathrm{~nm}$, which has nearly approached the applicability limit of the RRKM theory. This is because the validity of the RRKM theory is based on the assumption that the intramolecular vibrational relaxation is much faster than the bond dissociation or rearrangements. Similar results have been reported in refs $31-36$. The bond rupture rate constant $k_{6}$ is calculated employing the variational transition state geometry in which the dissociating $\mathrm{C}-\mathrm{H}$ bond was chosen as 2.4 and $3.0 \AA$; this has been proven to be sensible. ${ }^{33-35}$ As expected, the rate constants $k(E)$ obtained by the RRKM theory increase with $E$ or the wavelength changing from 193 to $157 \mathrm{~nm}$, and while the $k(E)$ of the HO will keep increasing, $k(E)$ for the $\mathrm{MO}$ will reach a limit because of bond dissociation energies. The rate constants within the MO approximation are much larger than those obtained with $\mathrm{HO}$ in the channel $k_{-1}$ and $k_{2}$, whereas the other cases show the opposite tendency. This is because $k(E)$ involves both $W^{\neq}(E-$ $E_{\mathrm{a}}^{\neq}$) and $\rho(E)$ and their dependence on $E$ can be different in different situations. The present results should still be considered as preliminary and can be improved by obtaining better anharmonic surfaces involved in the reactions.

\section{Conclusions}

In this paper, we have presented a systematic approach to treat the anharmonic effect on unimolecular reactions, including both canonical and microcanonical cases. An emphasis is placed on the demonstration of the combination of this approach with the use of the anharmonic potential surfaces obtained from ab initio calculations. For numerical demonstration, we have used the Morse potential to describe the anharmonic effect. We have shown that according to our approach both $W(E)$ and $\rho(E)$ can be determined from thermodynamic quantities. As a practical 
example for studying the anharmonic effect on unimolecular reactions, we chose to test ethylene photodissociation at 157 and $193 \mathrm{~nm}$; this has been carried out by the first principle calculations. The Morse anharmonicity model is employed for calculations of the number of states, density of states, and the rate constants using the RRKM theory. By comparing the harmonic and anharmonic results, the anharmonic effect is in most cases not negligible and increases with energy, the anharmonicity factor, and the size of the systems. As is to be expected, the anharmonic effect is in most cases significant and should be considered especially for the dissociation of less stable systems. For these systems, it can be expected that the HO approximation is not valid and not only the anharmonic effect but also the rotation-vibration coupling become very important, which can be treated by using the approach presented in this paper. It is hoped that the theoretical treatment developed in this paper can be applied to isomerization and dissociation of neutral and ionic clusters.

Acknowledgment. This work was supported in part by NSC (Taiwan) and Academia Sinica. A.M.M. thanks the Chemical Sciences, Geosciences and Biosciences Division, Office of Basic Energy Sciences, Office of Science of the U.S. Department of Energy, for financial support of this research (Grant DE-FG0204ER15570).

\section{References and Notes}

(1) Krems, R.; Nordholm, S. Z. Phys. Chem. 2000, 214, 1467.

(2) (a) Shen, D.; Pritchard, H. O. J. Chem. Soc. Faradav Trans. 1996, 92 (8), 1297. (b) Hobza, P.; Havlas, Z. Chem. Rev. 2000, 100, 4253 and references therein.

(3) (a) Tou, J. C.; Lin, S. H. J. Chem. Phys. 1968, 49, 4187. (b) Lin, S. H.; Eyring, H. J. Chem. Phvs. 1963, 39, 1577. (c) Lin, S. H.; Eyring, H. J. Chem. Phys. 1964, 43, 2153.

(4) (a) McDowell, S. A. C. J. Mol. Struct. Theochem 2006, 770, 119. (b) Bhuiyan, L. B.; Hase, W. L. J. Chem. Phys. 1983, 78, 5052.

(5) Peslherbe, G. H.; Hase, W. L. J. Chem. Phys. 1996, 105, 7432.

(6) (a) Stein, S. E.; Rabinovitch, B. S. J. Chem. Phys. 1973, 58, 2438

(b) Beyer, T.; Swinehart, D. F. Commun. Assoc. Comput. Machines 1973 , 16, 379. (c) Mills, I. M. Theoretical Chemistry; The Chemical Society: London, 1974; Vol. 1, Quantum Chemistry, p 110. (d) Schlag, E. W. Sandsmark, R. A. J. Chem. Phys. 1962, 37, 168.

(7) (a) Hase, W. L. Acc. Chem. Res. 1998, 31, 659. (b) Song, K.; Hase, W. J. Chem Phys. 1999, 110, 6198.

(8) Bagratashvilli, V. N.; Letokhov, V. S.; Makarov, A. A.; Ryabov,

E. A. Laser Chem. 1983, 1, 211.

(9) Mitra, S. S.; Bhattacharyya, S. S. J. Phys. B: At. Mol. Opt. Phys 1994, 27, 1773 .

(10) (a) Troe, J. Chem. Phys. 1995, 190, 381. (b) Troe, J. J. Phys. Chem. 1979, 83, 114. (c) Troe, J. J. Chem. Phys. 1983, 79, 6017. (d) Romanini, D.; Lehmann, K. K. J. Chem. Phvs. 1993, 98, 6437.

(11) (a) Forst, W.; Prãšil, Z. J. Chem. Phvs. 1970, 53, 3065. (b) Forst, W. Chem. Rev. 1971, 71, 339. (c) Forst, W. Theory of Unimolecular Reactions; Academic Press: New York, 1973.

(12) Hoare, M. R.; Ruijgrok, Th. W. J. Chem. Phys. 1970, 52, 113.

(13) Bates, J. R.; Taylor, H. S. J. Am. Chem. Soc. 1927, 49, 2439.

(14) Mooney, R. B.; Ludlam, E. B. Trans. Faraday Soc. 1929, 25, 442.

(15) LeRoy, D. J.; Steacie, E. W. R. J. Chem. Phys. 1941, 9, 829.

(16) LeRoy, D. J.; Steacie, E. W. R. J. Chem. Phys. 1942, 10, 676.

(17) Callear, A. B.; Cvetanovic, R. J. J. Chem. Phys. 1956, 24, 873.

(18) Sauer, M. C., Jr.; Dorfman, L. M. J. Chem. Phys. 1961, 35, 497.

(19) Okabe, H.; McNesby, J. R. J. Chem. Phys. 1962, 36, 601.

(20) Ausloos, P.; Gorden, R., Jr. J. Chem. Phys. 1962, 36, 5.

(21) Back, R. A.; Griffiths, D. W. L. J. Chem. Phys. 1967, 46, 4839

(22) Evleth, E. M.; Sevin, A. J. Am. Chem. Soc. 1981, 103, 7414.

(23) Raghavachari, K.; Frisch, M. J.; Pople, J. A.; Schleyer, R. R. Chem. Phys. Lett. 1982, 85, 145.
(24) Lin, J. J.; Hwang, D. W.; Lee, Y. T.; Yang, X. M. J. Chem. Phvs 1998, 109, 2979.

(25) Qi, F.; Sorkhabi, O.; Suits, A. G.; Chien, S. H.; Li, W. K. J. Am. Chem. Soc. 2001, 123, 148.

(26) Satyapal, S.; Johnston, G. W.; Bersohn, R.; Oref, I. J. Chem. Phvs. 1990, 93, 6398.

(27) Cromwell, E. F.; Stolow, A.; Vrakking, M. J. J.; Lee, Y. T. J. Chem. Phys. 1992, 97, 4029.

(28) Balko, B. A.; Zhang, J.; Lee, Y. T. J. Chem. Phys. 1992, 97, 935.

(29) Stolow, A.; Balko, B. A.; Cromwell, E. F.; Zhang, J.; Lee, Y. T. J. Photochem. Photobiol. A: Chem. 1992, 62, 285.

(30) Baer, T.; Hase, W. L. Unimolecular Reaction Dynamics: Theory and Experiments; Oxford University Press: New York, 1996.

(31) Mebel, A. M.; Chen, Y. T.; Lin, S. H. Chem. Phys. Lett. 1996, $258,53$.

(32) Mebel, A. M.; Chen, Y. T.; Lin, S. H. J. Chem. Phys. 1996, 105, 9007.

(33) Mebel, A. M.; Hayashi, M.; Lin, S. H. Chem. Phys. Lett. 1997, $174,281$.

(34) Hayashi, M.; Mebel, A. M.; Liang, K. K.; Lin, S. H. J. Chem. Phys. 1998, 108, 2044.

(35) Chang, A. H. H.; Mebel, A. M.; Yang, X. M.; Lin, S. H.; Lee, Y. T. Chem. Phys. Lett. 1998, 287, 301.

(36) Chang, A. H. H.; Hwang, D. W.; Yang, X. M.; Mebel, A. M.; Lin,

S. H.; Lee, Y. T. J. Chem. Phys. 1999, 110, 10810.

(37) Rice, O. K.; Ramsperger, H. C. J. Am. Chem. Soc. 1927, 49, 1617

(38) Kassel, L. S. J. Phys. Chem. 1928, 32, 1065.

(39) Eyring, H. J. Chem. Phys. 1935, 3, 107.

(40) (a) Marcus, R. A. J. Chem. Phys. 1952, 20, 359. (b) Marcus, R.

A.; Rice, O. K. J. Phvs. Colloid Chem. 1951, 55, 894.

(41) Wieder, G. M.; Marcus, R. A. J. Chem. Phys. 1962, 37, 1835.

(42) Lin, S. H.; Lau, K. H.; Richardson, W.; Volk, L.; Eyring, H. Proc. Natl. Acad. Sci. U.S.A. 1972, 69, 2778.

(43) (a) Miller, W. H. J. Am. Chem. Soc. 1979, 101, 23. (b) Faraday Discuss. Chem. Soc. 1991, 91, 479. (c) Kiermeier et al. Z. Naturforsch.

1987, 42a, 1399. (d) Neusser, H. J. J. Phys. Chem. 1989, 93, 3897.

(44) Gilbert, R. G.; Smith, S. C. Theory of Unimolecular and Recombination Reactions; Blackwell: Oxford, 1990.

(45) Bunker, D. L.; Hase, W. L. J. Chem. Phys. 1973, 59, 4621

(46) (a) Körtum, G.; Finckh, Z. F. Phys. Chem. 1942, B52, 263. (b) Ewing, G. E.; Sheng, De T. J. Phys. Chem. 1988, 92, 4063. (c) Berg, O.; Ewing, G. E. Surf. Sci. 1989, 220, 207.

(47) Truhlar, D. G.; Hase, W. L.; Hynes, J. T. J. Phys. Chem. 1983, 87, 2664.

(48) Quack, M.; Troe, J. Statistical Adiabatic Channel Model, in Encyclopedia of Computational Chemistry, 4; Ragué Schleyer, P. V., Allinger, N., Clark, T., Gasteiger, J., Kollman, P. A., Schaefer, H. F., III, Schreiner, P. R., Eds.; Wiley: New York, 1998; p 2708.

(49) Eyring, H.; Lin, S. H.; Lin, S. M. Basic Chemical Kinetics; WileyInterscience Publication: New York, 1980; Chapter 5,

(50) (a) Becke, A. D. J. Chem. Phvs. 1993, 98, 5648. (b) Lee, C.; Yang, W.; Parr, R. G. Phys. Rev. B 1988, 37, 785.

(51) (a) Purvis, G. L.; Bartlett, R. J. J. Chem. Phvs. 1982, 76, 1910. (b) Scuseria, G. E.; Janssen, C. L.; Schaefer, H. F., III. J. Chem. Phvs. 1988, 89, 7382. (c) Scuseria, G. E.; Schaefer, H. F., III. J. Chem. Phvs. 1989, 90, 3700. (d) Pople, J. A.; Head-Gordon, M.; Raghavachari, K. J. Chem. Phys. 1987, 87, 5968 .

(52) Frisch, M. J.; Trucks, G. W.; Schlegel, H. B.; Scuseria, G. E.; Robb, M. A.; Cheeseman, J. R.; Montgomery, J. A., Jr.; Vreven, T.; Kudin, K. N.; Burant, J. C.; Millam, J. M.; Iyengar, S. S.; Tomasi, J.; Barone, V.; Mennucci, B.; Cossi, M.; Scalmani, G.; Rega, N.; Petersson, G. A Nakatsuji, H.; Hada, M.; Ehara, M.; Toyota, K.; Fukuda, R.; Hasegawa, J.; Ishida, M.; Nakajima, T.; Honda, Y.; Kitao, O.; Nakai, H.; Klene, M.; Li, X.; Knox, J. E.; Hratchian, H. P.; Cross, J. B.; Bakken, V.; Adamo, C.; Jaramillo, J.; Gomperts, R.; Stratmann, R. E.; Yazyev, O.; Austin, A. J.; Cammi, R.; Pomelli, C.; Ochterski, J. W.; Ayala, P. Y.; Morokuma, K.; Voth, G. A.; Salvador, P.; Dannenberg, J. J.; Zakrzewski, V. G.; Dapprich, S.; Daniels, A. D.; Strain, M. C.; Farkas, O.; Malick, D. K.; Rabuck, A. D.; Raghavachari, K.; Foresman, J. B.; Ortiz, J. V.; Cui, Q.; Baboul, A. G.; Clifford, S.; Cioslowski, J.; Stefanov, B. B.; Liu, G.; Liashenko, A.; Piskorz, P.; Komaromi, I.; Martin, R. L.; Fox, D. J.; Keith, T.; Al-Laham, M. A.; Peng, C. Y.; Nanayakkara, A.; Challacombe, M.; Gill, P. M. W.; Johnson, B.; Chen, W.; Wong, M. W.; Gonzalez, C.; Pople, J. A. Gaussian 03, revision C.02; Gaussian, Inc.: Wallingford, CT, 2004 